\title{
TENSOLVE: A Software Package for Solving Systems of Nonlinear Equations and Nonlinear Least Squares Problems Using Tensor Methods
}

\author{
Ali Bouaricha" \\ Argonne National Laboratory \\ and \\ Robert B. Schnabel t \\ University of Colorado
}

This paper describes a modular software package for solving systems of nonlinear equations and nonlinear least squares problems, using a new class of methods called tensor methods. It is intended for small to medium-sized problems, say with up to 100 equations and unknowns, in cases where it is reasonable to calculate the Jacobian matrix or approximate it by finite differences at each iteration. The software allows the user to select between a tensor method and a standard method based upon a linear model. The tensor method models $F(x)$ by a quadratic model, where the second-order term is chosen so that the model is hardly more expensive to form, store, or solve than the standard linear model. Moreover, the software provides two different global strategies, a line search and a two-dimensional trust region approach. Test results indicate that, in general, tensord methods are significantly more efficient and robust than standard methods on small and medium-sized problems in iterations and function evaluations.

Categories and Subject Descriptors: G.1.5 [Numerical Analysis]: Roots of Nonlinear Equationssystems of equations; G.1.6 [Numerical Analysis]: Optimization-least squares methods; G.4 [Mathematics of Computing]: Mathematical Software

General Terms: Algorithms

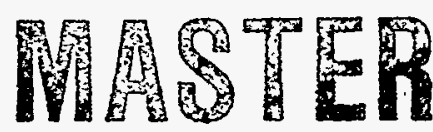

Additional Key Words and Phrases: tensor methods, nonlinear equations, nonlinear least squares, rank-deficient matrices

"Mathematios and Computer Science Division, Argonne National Laboratory, Argonne, Пlinois 60439, bouarich 9 mcs.anl.gov. Research supported in part by the Office of Scientific Computing, U.S. Department of Energy, nader Contract W-31-109-Eng-38.

'Department of Compater Science, University of Colorado, Boulder, Colorado 80309-0430, bobby9cs.colorado.edn. Research supported by AFOSR Grants No. AFOSR-90-0109 and F49620-94-1-0101, ARO Grants No. DAAL03-91-G-0151 and DAAB04-94-G-0228, and NSF Grant No. CCR-9101795. 


\section{DISCLAIMER}

Portions of this document may be illegible in electronic image products. Images are produced from the best available original document. 


\section{DISCLAIMER}

This report was prepared as an account of work sponsored by an agency of the United States Government. Neither the United States Government nor any agency thereof, nor any of their employees, make any warranty, express or implied, or assumes any legal liability or responsibility for the accuracy, completeness, or usefulness of any information, apparatus, product, or process disclosed, or represents that its use would not infringe privately owned rights. Reference herein to any specific commercial product, process, or service by trade name, trademark, manufacturer, or otherwise does not necessarily constitute or imply its endorsement, recommendation, or favoring by the United States Government or any agency thereof. The views and opinions of authors expressed herein do not necessarily state or reflect those of the United States Government or any agency thereof. 


\section{Introduction}

This paper describes a modular software package for solving systems of nonlinear equations of the form

$$
F: R^{n} \rightarrow R^{m}, \quad m \geq n,
$$

where $F$ is assumed to be at least once continuously differentiable, using a new class of methods called tensor methods. If $m$ is equal to $n$, the package solves the nonlinear equations problem, $F(x)=0$, while if $m$ is greater than $n$ it solves the nonlinear least squares problem, $\min _{x \in R^{n}}\|F(x)\|_{2}$.

Tensor methods base each iteration on a quadratic model of the nonlinear function,

$$
M\left(x_{c}+d\right)=F\left(x_{c}\right)+F^{\prime}\left(x_{c}\right) d+\frac{1}{2} T_{c} d d
$$

where $x_{c}$ is the current iterate, and $T_{c}$ is a three-dimensional object referred to as a tensor. No second derivative information is used in forming the tensor term $T_{c}$. Instead, $T_{c}$ is formed by asking the model to interpolate up to $\sqrt{n}$ past function values in a way that hardly increases the storage requirements or arithmetic cost per iteration over standard linear model based methods. The package also provides an option to use a method based on the standard linear model ((1.2) without the tensor term); it then performs a standard Newton method for nonlinear equations or Gauss-Newton method for nonlinear least squares. The global strategy used in either case can either be a line search strategy or a two-dimensional trust region method over the subspace spanned by the steepest descent direction and the tensor (or Newton/Gauss-Newton) step.

Required input to the package includes the dimensions $m$ and $n$ of the problem, where $m$ is the number of nonlinear equations and $n$ is the number of unknowns; a subroutine to evaluate the function $F(x)$; and an estimate $x_{0}$ of the solution $x_{*}$. The user may provide a code to calculate the Jacobian rather than having it computed by finite differences, may choose the standard method rather than the tensor method, and may specify various tolerances.

Upon completion, the program returns with an approximation $x_{p}$ to the solution $x_{*}$, the value of the sum of squares of the function $F\left(x_{p}\right)$, the value of the gradient $G\left(x_{p}\right)=F^{\prime}\left(x_{p}\right)^{T} F\left(x_{p}\right)$ of the function $\frac{1}{2}\|F(x)\|_{2}^{2}$ at $x_{p}$, and a flag specifying under which stopping condition the algorithm was terminated.

The tensor methods upon which this software package is based were originally introduced by Schnabel and Frank [11], for nonlinear equations. One main contribution of this paper is the provision and extensive testing of a software package incorporating these methods. In addition, the extension of these methods to nonlinear least squares, and the incorporation of a trust region strategy with tensor methods, are new contributions of this paper.

The remainder of this paper is organized as follows. In Section 2 we give a brief overview of tensor methods for nonlinear least squares problems (tensor methods for nonlinear equations can be regarded as a special case of these). In Section 3 we discuss the globally convergent modifications for tensor methods for systems of nonlinear equations and nonlinear least squares problems. Section 4 gives an overview of the key features and options provided by the software package. We then describe the user interface to the package in Section 5, which includes both a simplified default calling sequence and a longer calling sequence. In Section 6 we describe the meaning of the input, input-output, and output parameters for the package. Section 7 presents 
the default values provided by the package. A few implementation dependencies are described in Section 8. Section 9 gives an example of the use of the package. Finally, in Section 10 we summarize and discuss our experimental results using the package, with both line search and trust region strategies, on nonsingular and singular test problems.

\section{Brief Overview of Tensor Methods}

Tensor methods are general-purpose methods intended especially for problems where the Jacobian matrix at the solution is singular or ill-conditioned. The idea is to base each iteration upon a model that has more information than the standard linear model but is not appreciably more expensive to form, store, or solve. Specifically, each iteration is based upon a quadratic model (1.2) of the nonlinear function $F(x)$. The particular choice of the tensor term $T_{c} \in R^{m \times n \times n}$ causes the second-order term $T_{c} d d$ in (1.2) to have a simple and useful form. The tensor term is chosen to allow the model $M\left(x_{c}+d\right)$ to interpolate values of the function $F(x)$ at past iterates $x_{-k} ;$ that is, the model should satisfy

$$
F\left(x_{-k}\right)=F\left(x_{c}\right)+F^{\prime}\left(x_{c}\right) s_{k}+\frac{1}{2} T_{c} s_{k} s_{k}, \quad k=1, \ldots, p
$$

where

$$
s_{k}=x_{-k}-x_{c}, \quad k=1, \ldots, p .
$$

The past points $x_{-1}, \ldots, x_{-p}$ are selected so that the set of directions $\left\{s_{k}\right\}$ from $x_{c}$ to the selected points is strongly linearly independent; each direction $s_{k}$ is required to make an angle of at least 45 degrees with the subspace spanned by the previously selected past directions. The procedure of finding linearly independent directions is implemented easily by using a modified Gram-Schmidt algorithm, and usually results in $p=1$ or 2 .

After selecting the linearly independent past directions $s_{k}$, the tensor term is chosen by the procedure of Schnabel and Frank [11], which generalizes in a straightforward way to nonlinear least squares. $T_{c}$ is chosen to be the smallest matrix that satisfies the interpolation conditions (2.1); that is,

$$
\begin{gathered}
\min _{T_{c} \in R^{m \times n \times n}}\left\|T_{c}\right\|_{F} \\
\text { subject to } T_{c} s_{k} s_{k}=2\left(F\left(x_{-k}\right)-F\left(x_{c}\right)-F^{\prime}\left(x_{c}\right) s_{k}\right),
\end{gathered}
$$

where $\left\|T_{c}\right\|_{F}$, the Frobenius norm of $T_{c}$, is defined by

$$
\left\|T_{c}\right\|_{F}{ }^{2}=\sum_{i=1}^{m} \sum_{j=1}^{n} \sum_{k=1}^{n}\left(T_{c}[i, j, k]\right)^{2} .
$$

The solution to $(2.2)$ is the sum of $p$ rank-one tensors whose horizontal faces are symmetric:

$$
T_{c}=\sum_{k=1}^{p} a_{k} s_{k} s_{k},
$$

where $a_{k}$ is the $k$-th column of $A \in R^{m \times p}, A$ defined by $A=Z M^{-1}, Z$ is an $(m \times p)$ matrix whose columns are $Z_{j}=2\left(F\left(x_{-j}\right)-F\left(x_{c}\right)-F^{\prime}\left(x_{c}\right) s_{j}\right)$, and $M$ is a $(p \times p)$ matrix defined by $M(i, j)=\left(s_{i}{ }^{T} s_{j}\right)^{2}, 1 \leq i, j \leq p$. 
If we use the tensor term (2.4), the tensor model (1.2) becomes

$$
M\left(x_{c}+d\right)=F\left(x_{c}\right)+F^{\prime}\left(x_{c}\right) d+\frac{1}{2} \sum_{k=1}^{p} a_{k}\left\{d^{T} s_{k}\right\}^{2} .
$$

The simple form of the quadratic term in (2.5) is the key to being able to efficiently form, store, and solve the tensor model. The cost of forming the tensor term in the tensor model is $O(m n p) \leq O\left(m n^{1.5}\right)$ arithmetic operations, since $p \leq \sqrt{n}$, which is small in comparison with the $O\left(m n^{2}\right)$ cost per iteration of Gauss-Newton methods. The additional storage required is $4 p$ $m$-vectors, which is small in comparison with the storage for the Jacobian matrix.

Once the tensor model (2.5) is formed, a root of the tensor model is found. It is possible that no root exists; in this case a least squares solution of the model is found instead. Thus, in general, we solve the problem

$$
\text { find } d \in R^{n} \text { that minimizes }\left\|M\left(x_{c}+d\right)\right\|_{2} \text {. }
$$

A generalization of the process in Schnabel and Frank [11] shows that the solution to (2.6) can be reduced to the solution of a small number of quadratic equations, $m-n+q$ quadratic equations in $p$ unknowns, plus the solution of $n-q$ linear equations in $n-p$ unknowns. Here $q$ is equal to $p$ whenever $F^{\prime}\left(x_{c}\right)$ is nonsingular and usually when $\operatorname{rank}\left(F^{\prime}\left(x_{c}\right)\right) \geq n-p$; otherwise, $q$ is greater than $p$. Thus the system of linear equations is square or underdetermined, and the system of quadratic equations is equally determined or overdetermined. The main steps of the algorithm are the following:

1. An orthogonal transformation of the variable space is used to cause the $m$ equations in $n$ unknowns to be linear in $n-p$ variables, $\hat{d}_{1} \in R^{n-p}$, and quadratic only in the remaining $p$ variables, $\hat{d}_{2} \in R^{p}$.

2. An orthogonal transformation of the equations is used to eliminate the $n-p$ transformed linear variables from $n-q$ of the equations. The result is a system of $m-n+q$ quadratic equations in the $p$ unknowns, $\tilde{d}_{2}$, plus a system of $n-q$ equations in all the variables that is linear in the $n-p$ unknowns, $\tilde{d}_{1}$.

3. A nonlinear unconstrained optimization software package, UNCMIN [12], is used to minimize the $l_{2}$ norm of the $m-n+q$ quadratic equations in the $p$ unknowns, $\hat{d}_{2}$. (If $p=1$, this procedure is done analytically instead.)

4. The system of $n-q$ linear equations that is linear in the remaining $n-p$ unknowns is solved for $\tilde{d}_{1}$.

The arithmetic cost per iteration of the above process is the standard $O\left(m n^{2}\right)$ cost of a QR factorization of an $m \times n$ matrix, plus an additional $O(m n p) \leq O\left(m n^{1.5}\right)$ operations, plus the cost of using UNCMIN in Step 3 of the algorithm. The cost of using UNCMIN is expected to be $O\left(p^{4}\right) \leq O\left(n^{2}\right)$ operations, since each iteration requires $O\left(p^{3}\right)\left(O\left(p^{2} q\right)\right.$ when $\left.q>p\right)$ operations and a small multiple of $p$ iterations generally suffice. Thus, the total cost of the above algorithm is the $O\left(m n^{2}\right)$ cost of the standard method plus at most an additional cost of 
$O\left(m n^{1.5}\right)$ arithmetic operations. Note that in the case when $p=1$ and $q \geq 1$, the one-variable minimization problem is solved very inexpensively in closed form; this turns out to be the most common case in practice.

The Newton or Gauss-Newton step is computed inexpensively (in $O(m n p)$ operations) as a by-product of the tensor step solution. Using the tensor step and the Newton or Gauss-Newton step, the global portion of the algorithm determines the next iterate, as is described in the next section. The overall algorithm is summarized below.

Algorithm 2.1. An Iteration of the Tensor Method

Given $m, n, x_{c}, F\left(x_{c}\right)$

1. Calculate $F^{\prime}\left(x_{c}\right)$, and decide whether to stop, if not:

2. Select the past points to use in the tensor model from among the $\sqrt{n}$ most recent points.

3. Calculate the second-order term of the tensor model, $T_{c}$, so that the tensor model interpolates $F(x)$ at all the points selected in Step 2 .

4. Find the root of the tensor model, or its minimizer (in the $l_{2}$ norm) if it has no real root.

5. If $m>n$ or GLOBAL = tro-dimensional trust region then

5.1. Compute the standard step as a by-product of the tensor model solution.

5.2. Select the tensor or standard step using Algorithm 3.1 .

6. Select $x_{+}$using either a line search or a tro-dimensional trust region global strategy.

6.1. If GLOBAL = Iine search then

If $m>n$ perform Algorithm 3.3, where the search direction is

the step selected in Step 5.2

Else $\{m=n\}$ perform Algorithm 3.2.

ElseIf GLOBAL = tro-dimensional trust region then

Perform Algorith 3.4 using the model selected in Step 5.2.

7. Set $x_{c}-x_{+}, F\left(x_{c}\right) \leftarrow F\left(x_{+}\right)$, go to Step 1 .

The reader may refer to [1], [2], [6], and [11] for more details on tensor methods for nonlinear equations and nonlinear least squares problems. These papers give preliminary indications that tensor methods are more efficient and more robust computationally than standard methods, and show that tensor methods have a superior rate of convergence to Newton's method on nonlinear equations problems where $\operatorname{rank}\left\{F^{\prime}\left(x_{*}\right)\right\}=n-1$.

\section{Globally Convergent Modifications for Tensor Methods}

This section describes the global strategies in the tensor algorithm given above. As with all algorithms for nonlinear equations and optimization, purely local tensor methods may fail to converge if the initial guess is far away from the solution. To address this problem, two types of modifications are used in general, line search methods and trust region methods, and either may 
be best for a particular problem. For this reason, both of these global methods are included in our software package.

This section first describes the overall framework that is used in both the line search and trust region approaches for tensor methods. This framework involves a choice of whether to use the tensor step or the Newton/Gauss-Newton step as the basis for the global strategy at a given iteration. Next we briefly describe the line search that is used in the line search methods. Finally, we describe a new model trust region approach for tensor methods that is used in the trust region methods.

\subsection{Globally Convergent Framework for Tensor Methods for Nonlinear Least Squares}

Our computational experience has shown that when one is far from the solution, it is important to sometimes allow the global step to be based upon the Newton/Gauss-Newton step rather than the tensor step, and we have constructed heuristics to make this choice. Our experimentation has led to two different sets of heuristics, one that is used in both the line search and trust region methods for nonlinear least squares as well as the trust region method for nonlinear equations, and a second that is used in line search methods for nonlinear equations. They differ primarily in how much they bias the choice toward the tensor step. Both are constructed so that close to the solution, the tensor step is nearly always selected. This section gives these heuristics and the overall global frameworks that are based upon them.

Algorithm 3.1 gives the global framework that is used for nonlinear least squares and for trust region methods for nonlinear equations. In this framework, the Gauss-Newton step is chosen whenever the tensor step is not a descent direction, when the tensor step is a minimizer of the tensor model and does not provide enough decrease in the tensor model, or when the quadratic system of $m-n+q$ equations in $p$ unknowns cannot be solved by UNCMIN [12] within the iteration limit. Otherwise, the tensor step is chosen. In the definitions of $d_{t}$ and $M_{T}$, the Newton step and model are used for nonlinear equations, the Gauss-Newton step and model are used for nonlinear least squares.

Algorithm 3.1. Global Framevork for Tensor Hethods for Nonlinear Least Squares and for Trust Region Methods for Nonlinear Equations

Let

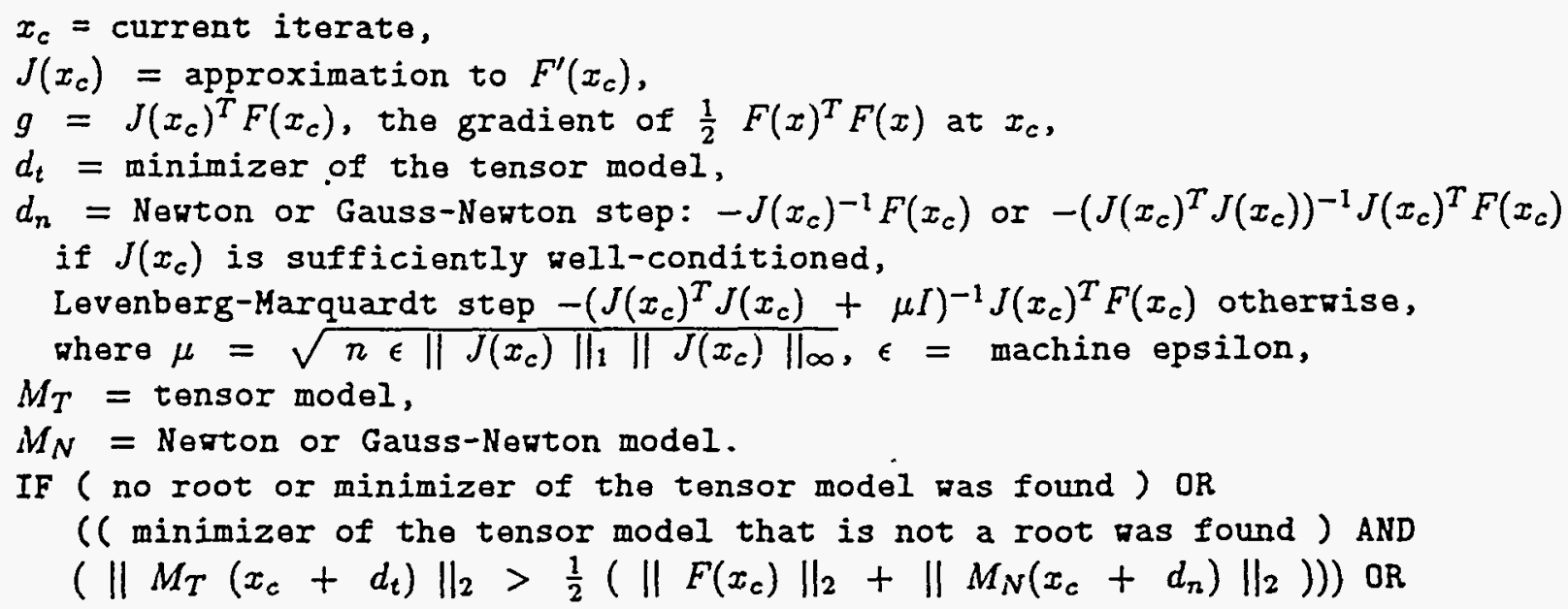




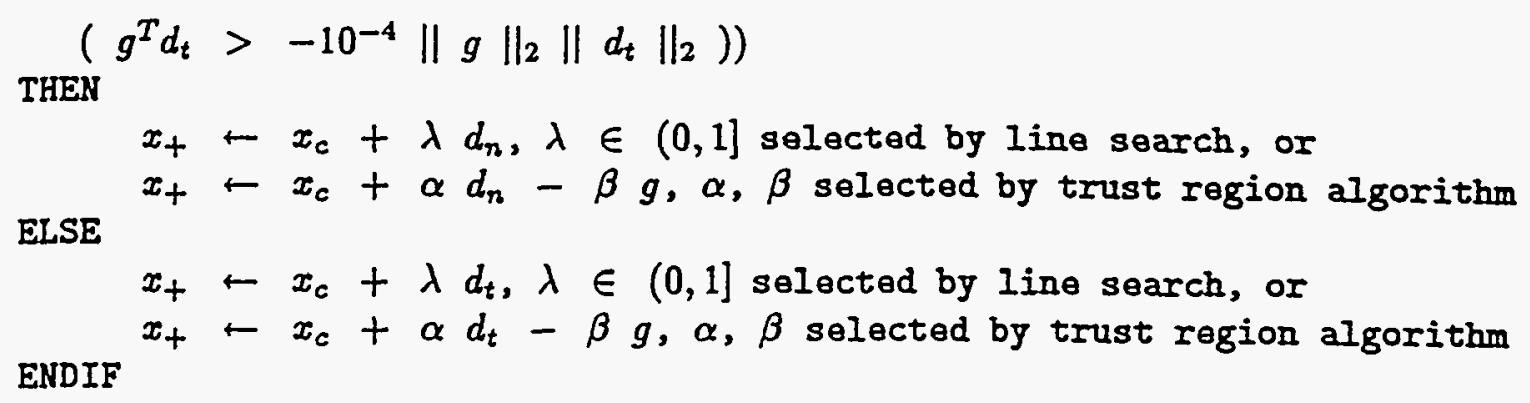

Algorithm 3.2 gives the global framework that is used in line search methods for nonlinear equations. Its main difference from Algorithm 3.1 is that it always tries the tensor step first, whether or not this step meets the descent or model decrease conditions of Algorithm 3.1. If $x_{c}+d_{t}$ provides enough decrease in $\|F(x)\|$, then it is used as the next iterate. If not, the strategy may tentatively compute global steps in both the Newton and the tensor directions. That is, the global step $x_{+}^{n}=x_{c}+\lambda d_{n}$ produced by a line search in the Newton direction $d_{n}$ is calculated. In addition, if $d_{t}$ is a descent direction, the global step $x_{t}^{t}=x_{c}+\lambda d_{t}$ produced by a line search in the tensor direction also is calculated. Finally, we select $x_{+}^{n}$ or $x_{+}^{t}$ depending on whichever has the lower function value. Thus, this strategy may involve one or more extra function evaluations when both line searches are performed.

\section{Algorithm 3.2. Global Framevork for Line Search Methods for Nonlinear Equations} Given $x_{c}, d_{n}, d_{t}, g$ as defined in Algorithm 3.1, and $\alpha=10^{-4}$. slope := $g^{T} d_{t}$

$$
\begin{aligned}
& f_{c}:=\frac{1}{2}\left\|F\left(x_{c}\right)\right\|_{2}{ }^{2} \\
& x_{+}^{t}:=x_{c}+d_{t} \\
& f_{+}:=\frac{1}{2}\left\|F\left(x_{+}^{t}\right)\right\|_{2}{ }^{2} \\
& \text { If } f_{+}<f_{c}+\alpha \cdot \min \{\text { slope, } 0\} \text { then } \\
& \quad \text { return } x_{+}=x_{+}^{t}
\end{aligned}
$$

Else

Find an acceptable $x_{+}^{n}$ in the Newton direction $d_{n}$, using Algorithm 3.3

comment. Test if the tensor step is sufficiently descent If $g^{T} d_{t} \geq-10^{-4}\|g\|_{2}\left\|d_{t}\right\|_{2}$ then

Else

$$
\text { return } x_{+}=x_{+}^{n}
$$

$$
\begin{aligned}
& \text { Find an acceptable } x_{+}^{t} \text { in the tensor direction } d_{t} \text {, } \\
& \text { using Algorithm } 3.3 \\
& \text { If }\left\|F\left(x_{+}^{n}\right)\right\|<\left\|F\left(x_{+}^{t}\right)\right\| \text { then } \\
& \text { return } x_{+}=x_{+}^{n} \\
& \text { Else return } x_{+}=x_{+}^{t}
\end{aligned}
$$

Endif

Endif 


\subsection{Global Framework for Line Search Methods for Nonlinear Equations}

The line search used in the global frameworks outlined above is a standard quadratic backtracking line search. It starts with $\lambda=1$ and then, if $x_{c}+d$ is not acceptable, reduces $\lambda$ until an acceptable $x_{c}+\lambda d$ is found, based upon a one-dimensional quadratic model of $F(x)^{T} F(x)$. Let us define

$$
\hat{f}(\lambda)=\frac{1}{2}\left\|F\left(x_{c}+\lambda d\right)\right\|_{2}^{2},
$$

the one-dimensional restriction of $f(x)=\frac{1}{2}\|F(x)\|_{2}^{2}$ to the line through $x_{c}$ in the direction $d$. If we need to backtrack, we use the values of $\hat{f}(0), \hat{f}^{\prime}(0)$, and $\hat{f}(\lambda)$ to model $\hat{f}$ and then take the value of $\lambda$ that minimizes this model as the next value of $\lambda$ in Algorithm 3.3 subject to restrictions on how much $\lambda$ can decrease at once (see, e.g., [4], pages 126-127 for more details). This results in the following algorithm.

Algorithm 3.3. Standard Quadratic Backtracking Line Search

$$
\begin{aligned}
& \text { Given } x_{c}, d, g=J\left(x_{c}\right)^{T} F\left(x_{c}\right) \text {, and } \alpha=10^{-4} . \\
& \text { slope }:=g^{T} d \\
& f_{c}:=\frac{1}{2}\left\|F\left(x_{c}\right)\right\|_{2}^{2} \\
& \lambda \quad:=1.0 \\
& x_{p}:=x_{c}+\lambda d \\
& f_{p}:=\frac{1}{2}\left\|F\left(x_{p}\right)\right\|_{2}^{2} \\
& \text { While } f_{p}>f_{c}+\alpha \cdot \lambda \cdot \text { slope do } \\
& \quad \lambda_{\text {lemp }}:=-\lambda \cdot \text { slope } /\left(2\left[f_{p}-f_{c}-\lambda \cdot \text { slope }\right]\right) \\
& \quad \lambda \quad:=\max \left\{\lambda_{l e m p}, \lambda / 10\right\} \\
& \quad x_{p}:=x_{c}+\lambda d \\
& f_{p}:=\frac{1}{2}\left\|F\left(x_{p}\right)\right\|_{2}^{2} \\
& \text { EndWhile }
\end{aligned}
$$

\subsection{Trust Region Tensor Methods for Nonlinear Equations and Nonlinear Least Squares}

Two computational methods-the locally constrained optimal (or "hook") method and the dogleg method-are generally used for approximately solving the trust region problem based on the standard model,

$$
\begin{gathered}
\text { minimize }\left\|F\left(x_{c}\right)+J\left(x_{c}\right) d\right\|_{2}{ }^{2} \\
\text { subject to }\|d\|_{2} \leq \delta_{c},
\end{gathered}
$$

where $\delta_{c}$ is the current trust region radius. When $\delta_{c}$ is shorter than the standard step, the locally constrained optimal method [8] finds a $\mu_{c}$ such that $\left\|d\left(\mu_{c}\right)\right\|_{2} \approx \delta_{c}$, where $d\left(\mu_{c}\right)=$ $-\left(J\left(x_{c}\right)^{T} J\left(x_{c}\right)+\mu I\right)^{-1} J\left(x_{c}\right)^{T} F\left(x_{c}\right)$. Then it takes $x_{+}=x_{c}+d\left(\mu_{c}\right)$. The dogleg method is a modification of the trust region algorithm introduced by Powell [10]. Rather than finding a point $x_{+}=x_{c}+d\left(\mu_{c}\right)$ on the curve $d\left(\mu_{c}\right)$ such that $\left\|x_{+}-x_{c}\right\| \approx \delta_{c}$, it approximates this curve by a piecewise linear function in the subspace spanned by the Newton direction and the steepest descent direction $-J\left(x_{c}\right)^{T} F\left(x_{c}\right)$, and takes $x_{+}$as the point on this piecewise curve for which $\left\|x_{+}-x_{c}\right\|=\delta_{c}$. (See, e.g., [4] for more details.) 
Unfortunately, these two methods are difficult to extend to the tensor model, because certain key properties do not generalize to this model. Trust region algorithms based on (3.1) are well defined because there is always a unique point $x_{+}$on the hookstep or dogleg curve such that $\left\|d\left(\mu_{c}\right)\right\|=\delta_{c}$. Additionally, the value of $\left\|F\left(x_{c}\right)+J\left(x_{c}\right) d\right\|_{2}{ }^{2}$ along these curves decreases monotonically from $x_{c}$ to $x_{+}^{n}$, where $x_{+}^{n}=x_{c}+d_{n}$, which makes the process reasonable. These properties do not extend to the fourth-order sum of squares of the tensor model, which may not be convex. Furthermore, the analogous curve to $d\left(\mu_{c}\right)$ is more expensive to compute. For these reasons, we consider a different trust region approach for our tensor methods.

The trust region approach that is used in this package is to solve a two-dimensional trust region problem over the subspace spanned by the steepest descent direction and the tensor (or standard) step. The main reasons that led us to adopt this approach are that it is easy to construct and is closely related to dogleg-type algorithms over the same subspace. In addition, the resultant step may be close to the optimal trust region step in practice. Byrd, Schnabel, and Shultz [3] have shown that for unconstrained optimization using a standard quadratic model, the analogous two-dimensional minimization approach produces nearly as much decrease in the quadratic model as the optimal trust region step in almost all cases.

The two-dimensional trust region approach for the tensor model computes an approximate solution to the exact trust region problem

$$
\begin{gathered}
\operatorname{minimize}\left\|F\left(x_{c}\right)+J\left(x_{c}\right) d+\frac{1}{2} \sum_{k=1}^{p} a_{k}\left\{d^{T} s_{k}\right\}^{2}\right\|_{2}^{2} \\
\text { subject to }\|d\|_{2} \leq \delta_{c},
\end{gathered}
$$

by performing a two-dimensional minimization

$$
\begin{gathered}
\text { minimize }\left\|F\left(x_{c}\right)+J\left(x_{c}\right) d+\frac{1}{2} \sum_{k=1}^{p} a_{k}\left\{d^{T} s_{k}\right\}^{2}\right\|_{2}^{2} \\
\text { subject to }\|d\|_{2} \leq \delta_{c}, \quad d \in\left[d_{t}, g_{s}\right],
\end{gathered}
$$

where $d_{t}$ and $g_{s}$ are the tensor step and the steepest descent direction, respectively, and $\delta_{c}$ is the trust region radius. This approach always produces a step that reduces the quadratic model by at least as much as a dogleg-type algorithm, which minimizes the model over a piecewise linear curve in the same subspace. When Algorithm 3.1 chooses the Newton or Gauss-Newton step, we instead solve the variant of (3.3) where $d_{t}$ is replaced by $d_{n}$ and the quadratic term in the model is omitted.

Before we give the complete two-dimensional trust region algorithm for tensor methods, we show how to convert the problem (3.3) into an unconstrained minimization problem in one variable. This transformation is the key to solving (3.3) efficiently. First, we form an orthonormal basis for the two-dimensional subspace by performing the projection

$$
\tilde{g}_{s}=g_{s}-d_{t} \frac{g_{s}^{T} d_{t}}{d_{t}^{T} d_{t}}
$$

and normalizing $\hat{g}_{s}$ and $d_{t}$ to obtain

$$
\tilde{d}_{t}=\frac{d_{t}}{\left\|d_{t}\right\|_{2}}, \quad \tilde{g}_{s}=\frac{\hat{g}_{s}}{\left\|\hat{g}_{s}\right\|_{2}}
$$


Since $d$ is in the subspace spanned by $\tilde{d}_{t}$ and $\tilde{g}_{s}$, it can be written as

$$
d=\alpha \tilde{d}_{t}+\beta \tilde{g}_{s}, \quad \alpha, \beta \in \Re .
$$

If we square the $l_{2}$ norm of this expression for $d$ and set it to $\delta_{c}^{2}$, we obtain the following equation for $\beta$ as a function of $\alpha$ :

$$
\beta=\sqrt{\delta_{c}^{2}-\alpha^{2}}
$$

Substituting this expression for $\beta$ into (3.6) and then the resulting $d$ into (3.3) yields the global minimization problem in the one variable $\alpha$,

$\left.\operatorname{minimize} \| F\left(x_{c}\right)+\alpha J\left(x_{c}\right) \tilde{d}_{t}+\sqrt{\delta_{c}^{2}-\alpha^{2}} J\left(x_{c}\right) \tilde{g}_{s}+\frac{1}{2} \sum_{k=1}^{p} \dot{a}_{k}\left(\alpha s_{k}^{T} \tilde{d}_{t}+\sqrt{\delta_{c}^{2}-\alpha^{2} s_{k}^{T}} \tilde{g}_{s}\right)^{2}\right) \|_{2}{ }^{2}$

where $-\delta_{c}<\alpha<\delta_{c}$. Thus, problems (3.7) and (3.3) are equivalent.

We use the same procedure to convert the problem

$$
\begin{gathered}
\text { minimize }\left\|F\left(x_{c}\right)+J\left(x_{c}\right) d\right\|_{2}{ }^{2} \\
\text { subject to }\|d\|_{2} \leq \delta_{c}, \quad d \in\left[d_{n}, g\right]
\end{gathered}
$$

- to the equivalent global minimization problem in the one variable $\alpha$,

$$
\text { minimize }\left\|F\left(x_{c}\right)+\alpha J\left(x_{c}\right) \tilde{d}_{n}+\sqrt{\delta_{c}^{2}-\alpha^{2}} J\left(x_{c}\right) \tilde{g}_{s}\right\|_{2}^{2},
$$

where $-\delta_{c}<\alpha<\delta_{c}$.

The two-dimensional trust region method for tensor methods is given in the following algorithm.

Algorithm 3.4. Tro-Dimensional Trust Region for Tensor Methods

Given $x_{c}, d_{n}, d_{t}$ as defined in Algorithm 3.1.

Let $g_{0}=-J\left(x_{c}\right)^{T} F\left(x_{c}\right)$, the steepest descent direction;

$\delta_{c}$ the current trust region radius;

$\tilde{d}_{t}$ and $\tilde{g}_{s}$ given by $(3.5)$;

$\bar{d}_{n}$ obtained in an analogous way to $\bar{d}_{t}$

by applying transformations (3.4) and (3.5) to $d_{n}$.

If tensor model selected then

Solve problem (3.7)

$d=\alpha_{*} \tilde{d}_{t}+\tilde{g}_{s} \sqrt{\delta_{c}^{2}-\alpha_{*}^{2}}$

where $\alpha_{*}$ is the global minimizer of (3.7)

Else \{ standard model selected \}

Solve problem (3.9)

$d=\alpha_{*} \tilde{d}_{n}+\tilde{g}_{s} \sqrt{\delta_{c}^{2}-\alpha_{*}^{2}}$

where $\alpha_{*}$ is the global minimizer of (3.9) 


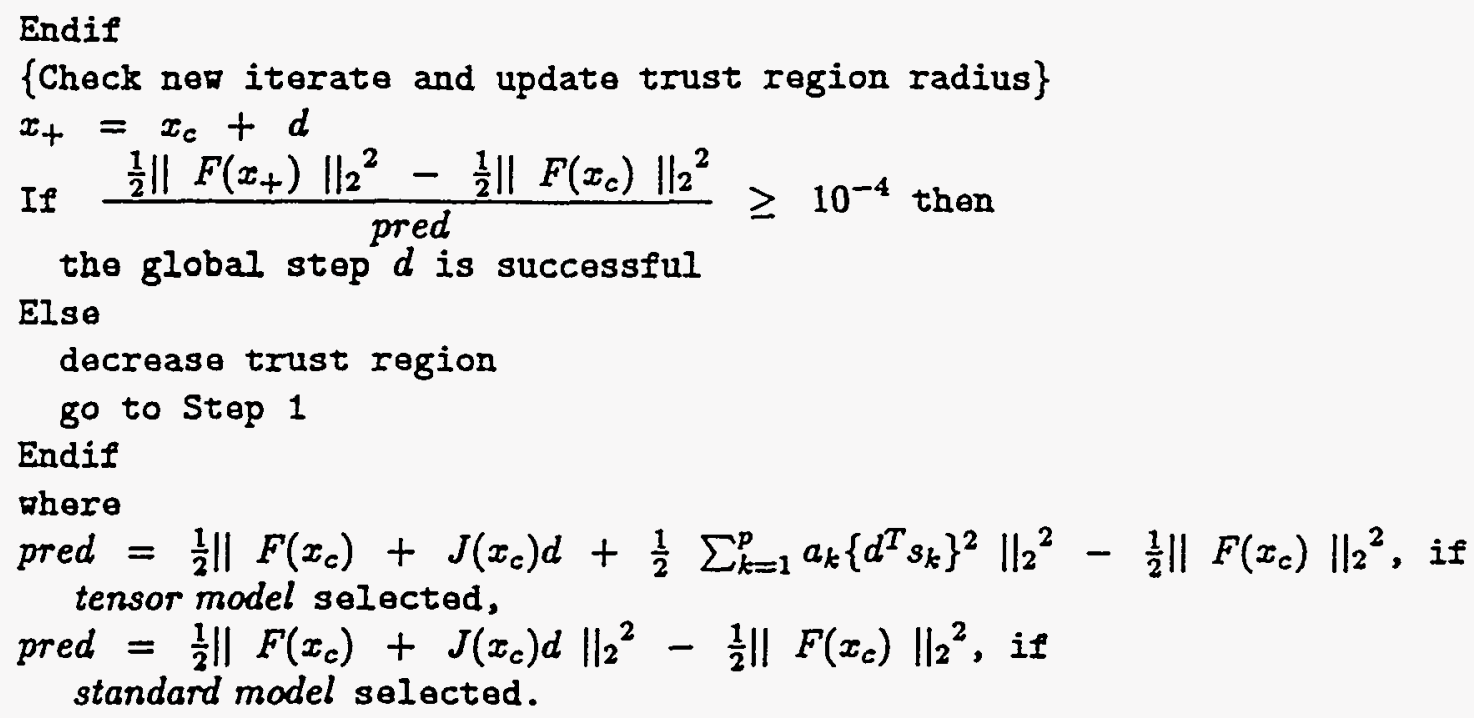

The methods used for adjusting the trust radius during and between steps are given in Algorithm A6.4.5 [9, p. 338]. The initial trust radius can be supplied by the user; if not, it is set to the length of the initial Cauchy step. Our software solves the one variable global optimization problem by a straightforward partitioning scheme described in [2].

\section{Overview of the Software Package}

This section summarizes the key features of the software package.

The user has the option to solve systems of nonlinear equations or nonlinear least squares problems. In either case, the required input for the software is the number of equations $M$, the number of variables $\mathrm{N}$, the function FVEC that computes $F(x)$, and an initial guess $X_{0}$. If $M=$ $N$, the problem is nonlinear equations; if $M>N$ it is nonlinear least squares. The user does not have to set a flag differentiating between the two problems.

Two methods of calling the package are provided. In the short version, the user supplies only the above information, and default values of all other options and parameters are used. (These include the use of the tensor rather than the standard method, the use of the line search global strategy, and the calculation of the Jacobian by finite differences). In the other method for calling the package. the user may override any default values of the package options and parameters.

The package allows the user to use the tensor method or the standard Newton or GaussNewton method. METHOD $=1$ specifies the tensor method and is the default value. If the flag METHOD is set to 0 , the package will use the standard method.

Two global strategies are implemented in the software package, a line search method, and a two-dimensional trust region method over the subspace spanned by the steepest descent direction and the tensor (or Newton/Gauss-Newton) step. The global strategy may be specified using the parameter GLOBAL. GLOBAL = 0 is the default and specifies the line search. GLOBAL $=1$ specifies the trust region.

The user may supply an analytic routine to evaluate the Jacobian matrix. If it is not supplied, the package computes the Jacobian by finite differences. The finite difference routine 
is described in detail by Dennis and Schnabel [4]. The parameter JACFLG specifies whether an analytic Jacobian has been provided. The default value, which specifies finite differences, is JACFLG $=0$. When the analytic Jacobian is supplied, the user has the option of checking the supplied analytic routine against the package's finite difference routine; if MSG is set to 2 modulo 4 , the package will not check the analytic Jacobian against the finite difference one; otherwise it will.

Scaling information for the variables and/or the functions may be supplied by the user. The software package is coded so that if the user inputs the typical magnitude typ $x_{i}$ of each component of $x$ and/or the typical magnitude typ $f_{i}$ of each component of the function $F$, the performance of the package is equivalent to what would result from redefining the independent variable $x$ in the user's function and the components of the function $F$ with

$$
\text { xscaled }=\left[\begin{array}{lll}
1 / \text { typx }_{1} & & \\
& \cdot \cdot \cdot & \\
& & \\
& & 1 / \text { typ }_{n}
\end{array}\right] \cdot x
$$

and/or

$$
\text { Fscaled }=\left[\begin{array}{lll}
1 / \operatorname{typ}_{1} & & \\
& \cdot \cdot \cdot \\
& & \\
& & 1 / \text { typ }_{\mathrm{m}}
\end{array}\right] \cdot F
$$

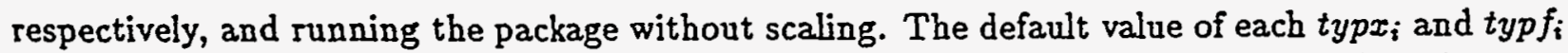
is 1 (i.e., no scaling). Scaling is often important to use for problems in which there is great variation in the magnitudes of individual variables and/or function components.

The package includes a module INCHK that examines the input parameters for illegal entries and consistency. Certain illegal or inconsistent entries are reset to default values by this module, while other illegal entries cause the package to terminate. Details are given in the parameter listing in Section 6.

The standard (default) output from this package consists of printing the input parameters; the final results, and the stopping condition. The printed input parameters are those used by the algorithm and hence include any corrections made by the module INCHK. The program will provide an error message if it terminates as a result of input errors. The printed results include a message indicating the reason for termination, an approximation $x_{p}$ to the solution $x_{*}$, the value of the sum of squares of the function $F\left(x_{p}\right)$, and the gradient vector $G\left(x_{p}\right)=F^{\prime}\left(x_{p}\right)^{T} F\left(x_{p}\right)$ of the function $\frac{1}{2}\|F(x)\|_{2}{ }^{2}$ at $x_{p}$. The package provides an additional means for controlling the 
output by means of the variable MSG, described in Section 6. The user may suppress all output or may print the intermediate iterations results in addition to the standard output.

\section{Interfaces and Usage}

Two interfaces are provided with the system. NONLQO requires the user to provide only the dimensions $M$ and $N$ of the problem, a subroutine to evaluate the function $F$, and a starting vector $X_{0}$ (as well as three work arrays and their dimensions). NONLQ requires the user to supply all parameters. However, the user may specify selected parameters only by first invoking the subroutine DFAULT, which sets all parameters to their default values, and then overriding only the desired values. This is the normal usage of NONLQ.

The two calling sequences are as follows.

1. CALL NONLQO (NRH, NRH, HC, XO, K, N, WRKUNC, WRKNEM, WRKNEN, FVEC, MSG, XP, FP, GP, TERHCD)

2. CALL DEAULT( $M, N$, ITNLIM, JACFLG, GRADTL, STEPTL, FTOL, METHOD, GLOBAL, STEPMX, DLT, TYPX, TYPF, IPR, MSG)

C USER OVERRIDES SPECIFIC DEFAULT VALUES PARARETERS, E.G.

$\begin{aligned} \text { GRADTL } & =1.0 \mathrm{D}-6 \\ \text { STEPTL } & =1.0 \mathrm{D}-7 \\ \text { FTOL } & =1.0 \mathrm{D}-10 \\ \text { JACFLG } & =1\end{aligned}$

CALL NONLQ (NRM, NRN, NC, XO, M, N, TYPX, TYPF, ITNLIM, JACFLG, GRADTL, STEPTL, FTOL, METHOD, GLOBAL, STEPMX, DLT, IPR, WRKUNC, WRKNEM, WRKNEN, ANJA, FVEC, MSG, XP, FP, GP, TERMCD)

\section{Parameters and Default Values}

The parameters employed with the calling sequences of Section 5 are fully described here. NONLQO uses only those parameters that are preceded by an asterisk. When it is noted that module DFAULT returns a given value, this is the value employed by interface NONLQO. The user may override the default value by utilizing NONLQ as shown above.

Following each variable name in the list below appears a one- or a two-headed arrow symbol of the form $\rightarrow,-$, and $\longrightarrow$. These symbols signify that the variable is for input, output, and input-output, respectively.

The symbol $\epsilon$ in some parts of this section designates the machine epsilon (see Section 8 ).

*NRM $\rightarrow:$ A positive integer specifying the row dimension of the work array WRKNEM in the user's calling program. NRM must satisfy the relation $N R M \geq M+N$; if not, the program will abort. The provision of this variable, NRN, and NC (below) allows the user the flexibility of solving several 
problems with different values of $M$ and $N$ one after the other, with the same work arrays.

*NRN $\rightarrow$ : A positive integer specifying the row dimension of the work array WRKNEN in the user's calling program. NRN must satisfy the relation NRN $\geq N$; if not, the program will abort.

*NC $\rightarrow:$ A positive integer specifying the row dimension of the work array WRKUNC in the user's calling program. NC must satisfy the relation NC $\geq\lceil\sqrt{N}\rceil$; if not, the program will abort.

$* X 0 \rightarrow:$ An array of length $N$ that contains an initial estimate of the solution $x *$.

$* \mathrm{M} \rightarrow:$ A positive integer specifying the number of nonlinear equations. The program will abort if $\mathrm{K} \leq \mathrm{0}$.

$* \mathrm{~N} \rightarrow$ : A positive integer specifying the number of variables in the problem. The program will abort if $\mathbb{N} \leq 0$.

TYPX $\rightarrow:$ An array of length $N$ in which the typical size of the components of $X$ is specified. The typical component sizes should be positive real scalars. If a negative value is specified, its absolute value will be used. If 0 . is specified, 1 . will be used. This vector is used by the package to determine the scaling matrix, $D_{x}$. Although the package may work reasonably well in a large number of instances without scaling, it may fail when the components of $x_{*}$ are of radically different magnitude and scaling is not invoked. If the sizes of the parameters are known to differ by many orders of magnitude, then the scale vector TYPX should definitely be used. For example, if it is anticipated that the range of values for the iterates $x_{k}$ would be

$$
\begin{aligned}
& x_{1} \in\left[-10^{10}, 10^{10}\right] \\
& x_{2} \in\left[-10^{2}, 10^{4}\right] \\
& x_{3} \in\left[-6 \times 10^{-6}, 9 \times 10^{-6}\right]
\end{aligned}
$$

then an appropriate choice would be TYPX $=(1.0 \mathrm{D} 10,1.0 \mathrm{D} 3,7.0 \mathrm{D}-6)$. Module DFAULT returns $\operatorname{TYPX}=(1.0 \mathrm{DO}, \ldots, 1.0 \mathrm{DO})$.

TYPF $\rightarrow$ : An array of length $M$ in which the typical size of the components of $F$ is specified. The typical component sizes should be positive real scalars. If a negative value is specified, its absolute value will be used. If 0 . is specified, 1 . will be used. This vector is used by the package to determine the scaling matrix $D_{F}$. TYPF should be chosen so that all the components of $D_{F}(x)$ have similar typical magnitudes at points not too near a root, and should be chosen in conjunction with FTOL. It is important to supply values of TYPF when the magnitudes of the components of $F(x)$ are expected to be very different; in this case, the program may work better with good scaling information than with $D_{F}=I$. If the magnitudes of the components of $F(x)$ are similar, the choice $D_{F}=I$ suffices. Module DFAULT returns TYPF $=(1.0 \mathrm{D} 0, \ldots, 1.0 \mathrm{DO})$.

ITNLIM $\rightarrow:$ Positive integer specifying the maximum number of iterations to be performed before the program is terminated. Module DFAUTT returns ITNLIM $=150$. If the user specifies ITNLIM $\leq 0$, the module INCHK will supply the value 150 . 
JACFLG $\rightarrow$ : Integer flag designating whether or not an analytic Jacobian has been supplied by the user.

JACFLG $=0:$ No analytic Jacobian supplied.

JACFLG $=1:$ Analytic Jacobian supplied.

When JACFLG $=0$, the Jacobian is obtained by finite differences. The module DFAULT returns the value 0 . If the user specifies an illegal value, the module INCHK will supply the value 0 .

GRADTL $\rightarrow$ : Positive scalar giving the tolerance at which the scaled gradient of $f(x)=\frac{1}{2} F(x)^{r} F(x)$ is considered close enough to zero to terminate the algorithm. The scaled gradient is a measure of the relative change in $F$ in each direction $x_{i}$ divided by the relative change in $x_{i}$. More precisely, the test used by the program is

$$
\max _{i}\left\{\frac{|\nabla f(x)|_{i} \max \left\{\left|x_{i}\right|, \text { TYPX }_{i}\right\}}{\max \{F n o r m, n / 2\}}\right\} \leq \text { GRADTL. }
$$

Here $\nabla f(x)=J(x)^{T} D_{F}^{2} F(x)$, and $F$ norm $=\frac{1}{2}\left\|D_{F} F(x)\right\|_{2}{ }^{2}$ where $D_{F}=\operatorname{diag}\left(1 / \operatorname{TYPF}_{1}, \ldots, 1 /\right.$ TYPF $\left._{m}\right)$. The module DFAULT returns the value $\epsilon^{1 / 3}$. If the user specifies a negative value, the module INCHK will supply the value $\epsilon^{1 / 3}$.

STEPTL $\rightarrow$ : A positive scalar providing the minimum allowable relative step length. STEPTL should be at least as small as $10^{-d}$, where $d$ is the number of accurate digits the user desires in the solution $x_{\text {. }}$. The actual test used is

$$
\max _{i}\left\{\frac{\left|x_{i}^{k}-x_{i}^{k-1}\right|}{\max \left\{\left|x_{i}^{k}, \operatorname{TYPX} x_{i}\right|\right\}}\right\} \leq \text { STEPTL. }
$$

The program may terminate prematurely if STEPTL is too large. Module DFAULT returns the value $\epsilon^{2 / 3}$. If the user specifies a negative value, the module INCHK will supply the value $\epsilon^{2 / 3}$.

FTOL $\rightarrow$ : A positive scalar giving the tolerance at which the scaled function $D_{F} F(x)$ is considered close enough to zero to terminate the algorithm. The program is halted if $\left\|D_{F} F(x)\right\|_{\infty}$ is $\leq$ FTOL. This is the primary stopping condition for nonlinear equations; the values of TYPF and FTOL should be chosen so that this test reflects the user's idea of what constitutes a solution to the problem. The module DFAULT returns the value $\epsilon^{2 / 3}$. If the user specifies a negative value, the module INCHK will supply the value $\epsilon^{2 / 3}$.

METHOD $\rightarrow:$ An integer flag designating which method to use.

METHOD $=0:$ Newton or Gauss-Newton algorithm is used.

METHOD $=1:$ Tensor algorithm is used.

Module DFAULT returns value 1 . If the user specifies an illegal value, module INCHK will reset METHOD to 1 , and execution will continue.

GLOBAL $\rightarrow:$ An integer flag designating which global strategy to use. GLOBAL $=0:$ Line search is used. 
GLOBAL $=1:$ Two-dimensional trust region is used.

Module DFAULT returns value of 0 . If the user specifies an illegal value, module INCHK will reset GLOBAL to 0 , and execution will continue.

STEPMX $\rightarrow:$ A positive scalar providing the maximum allowable scaled step length $\left\|D_{x}\left(x_{+}-x_{c}\right)\right\|_{2}$, where $D_{x}=\operatorname{diag}\left(1 / \operatorname{TYPX}_{1}, \ldots, 1 / \operatorname{TYPX}_{n}\right)$. STEPKX is used to prevent steps that would cause the nonlinear equations problem to overflow, and to prevent the algorithm from leaving the area of interest in parameter space. STEPMX should be chosen small enough to prevent these occurrences but should be larger than any anticipated "reasonable" step. Module DFAULT returns the value STEPMX $=10^{3}$. If the user specifies a nonpositive value, module INCHK sets STEPMX to $10^{3}$.

DLT $\rightarrow$ : A positive scalar giving the initial trust region radius. When the line search strategy is used, this parameter is ignored. For the trust region algorithm, if DLT is supplied, its value should reflect what the user considers a maximum reasonable scaled step length at the first iteration. If DLT is not supplied ( $D L T=-1.0$ ), the routine uses the length of the Cauchy step at the initial iterate instead. The module DFAULI returns the value -1.0 . If the user specifies a nonpositive value, module INCHK sets $\mathrm{DLT}=-1.0$.

IPR $\rightarrow:$ The unit on which the package outputs information. DFAULT returns the value 6 , which is the standard Fortran unit for the printer.

*HRKUNC $\rightarrow$ : Workspace used by UNCMIN. The user must declare this array to have dimensions at least $\mathrm{NC} \times(2[\sqrt{\mathrm{N}}\rceil+4)$ in the calling routine; if not, the program will abort.

*WRKNEM $\rightarrow$ : Workspace used to store the Jacobian matrix, the function values matrix FV, the tensor matrix ANLS, and working vectors. The user must declare this array to have dimensions at least NRMX $\times(N+2[\sqrt{N}\rceil+11)$ in the calling routine; if not, the program will abort.

*WRKNEN $\rightarrow$ : Workspace used to store the matrix $S$ of previous directions, the matrix SHAT of linearly independent directions, and working vectors. The user must declare this array to have dimensions at least $N R N \times(2\lceil\sqrt{\mathrm{N}}\rceil+13)$ in the calling routine; if not, the program will abort.

ANJA $\rightarrow$ : The name of a user-supplied subroutine that evaluates the first derivative (Jacobian) of the function $F(x)$. The subroutine must be declared EXTERNAL in the user's program and must conform to the usage

$$
\text { CALL ANJA(JAC, } X, N R M, N) \text {, }
$$

where $X$ is a vector of length $N$ and the 2 -dimensional array JAC is the analytic Jacobian of $F$ at $X$. When using the interface NONLQ, if no analytic Jacobian is supplied (JACFLG $=0$ ), the user must use the dummy name FDAJA as the value of this parameter.

*FVEC $\rightarrow$ : The name of a user-supplied subroutine that evaluates the function $F$ at an arbitrary vector $X$. The subroutine must be declared EXTERNAL in the user's calling program and must conform to the usage 
CALL FVEC $(X, F, M, N)$,

where $X$ is a vector of length $N$ and $F$ is a vector of length $M$. The subroutine must not alter the values of $X$.

*MSG $\rightarrow:$ An integer variable that the user may set on input to inhibit certain automatic checks or to override certain default characteristics of the package. (In the short call it should be set to 0 on input.) There are four "message" features that can be used individually or in combination as discussed below.

MSG = $0:$ Values of input parameters, final results, and termination code are printed.

MSG = 2 : Do not check user's analytic Jacobian routine against its finite difference estimate. This may be necessary if the user knows the Jacobian is properly coded, but the program aborts because the comparative tolerance is too tight. Do not use MSG $=2$ if the analytic Jacobian is not supplied.

MSG $=4$ : Suppress printing of the input state, the final results, and the stopping condition.

MSG = $8:$ Print the intermediate results; that is, the input state, each iteration including the current iterate $x_{k}, \frac{1}{2}\left\|D_{F} F\left(x_{k}\right)\right\|_{2}{ }^{2}$, and $\nabla f(x)=J(x)^{T} D_{F}{ }^{2} F(x)$, and the final results including the stopping conditions.

The user may specify a combination of features by setting MSG to the sum of the individual components. The module DFAULT returns a value of 0 . On output, if the program has terminated because of erroneous input, MSG contains an error code indicating the reason.

MSG $=0:$ No error.

MSG $=-1:$ Mlegal dimension, $N R M<M+M$.

MSG $=-2:$ Mlegal dimension, NRN $<N$.

MSG $=-3$ : Mlegal dimension, NC $<\lceil\sqrt{\mathrm{N}}]$.

MSG $=-4:$ Illegal dimension, $M \leq 0$.

MSG $=-5:$ \#legal dimension, $N \leq 0$.

MSG $=-6:$ Program asked to override check of analytic Jacobian against finite difference estimate, but routine ANJA not supplied (incompatible input).

MSG $=-7$ : Probable coding error in the user's analytic Jacobian routine ANJA. Analytic and finite difference Jacobian do not agree within the assigned tolerance.

*XP-: An array of length $N$ containing the best approximation to the solution $x$ * upon return. (If the algorithm has not converged, the final iterate is returned).

*FP-: An array of length $M$ containing the function value $F(X P)$.

*GP-: An array of length $N$ containing the gradient of the function $\frac{1}{2}\|F(x)\|_{2}^{2}$ at $X P$.

*TERMCD $\leftarrow:$ An integer that specifies the reason the algorithm was terminated.

TERMCD = $0:$ No termination criterion satisfied (occurs if package terminates because of illegal input).

TERMCD = 1 : function tolerance reached. Current iteration is probably solution.

TERMCD $=2:$ gradient tolerance reached. For nonlinear least squares, current iteration is prob- 
ably solution; for nonlinear equations, this could either be solution or local minimizer.

TERMCD $=3:$ Successive iterates within tolerance. Current iterate may be solution, or algorithm may have bogged down away from solution.

TERMCD $=4:$ Last global step failed to locate a point lower than XP. It is likely that either XP is an approximate solution of the problem or STEPTL is too large.

TERMCD $=5:$ Iteration limit exceeded.

\section{Summary of Default Values}

The following parameters are returned by the module DFAULT:

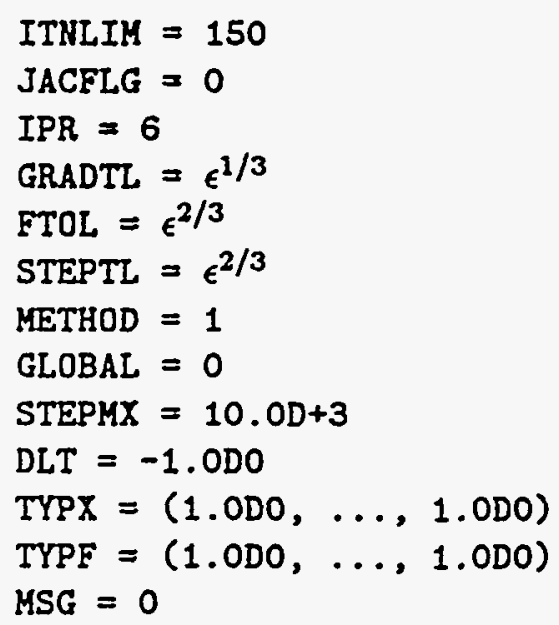

\section{Implementation Details}

This program package has been coded in Fortran 77 using double precision. It consists of approximately 8700 lines of code, of which 3400 lines are the software package UNCMIN [12] which has been designed to solve the unconstrained nonlinear optimization problem, and about $25 \%$ are comments. The total data storage required is about $M \times(N+2 \sqrt{N})+N \times(N+4 \sqrt{N})$ double-precision numbers. The program was developed and tested on a Sun 4 computer in the Computer Science Department at the University of Colorado at Boulder.

There is one machine dependency. The machine epsilon is calculated by the package and used in several places, including finite differences stepsizes and stopping criteria. On some computers, the returned value may be incorrect because of compiler optimizations. The user may wish to check the computer value of the machine epsilon and, if it is incorrect, replace the code in the subroutine MACEPS with the following statement.

$$
\text { EPS = correct value of machine epsilon }
$$

\section{Example of Use}

In the example code shown below in Figure 1, we first call the default routine DFAULT which returns with the default values, then override the values of GRADTL, FTOL, and STEPTL. Then we 


\begin{tabular}{|c|c|c|}
\hline NESLV & \multicolumn{2}{|l|}{ TYPICAL $X$} \\
\hline NESLV & \multirow{2}{*}{\multicolumn{2}{|c|}{$\begin{array}{l}0.1000000000000 D+010.1000000000000 D+01 \\
\text { DIAGONAL SCALING MATRIX FOR X }\end{array}$}} \\
\hline NESLV & & \\
\hline NESLV & \multicolumn{2}{|c|}{$0.1000000000000 D+01 \quad 0.1000000000000 D+01$} \\
\hline NESLV & \multicolumn{2}{|l|}{ TYPICAL $F$} \\
\hline NESLV & $0.1000000000000 D+01$ & $0.1000000000000 D+01$ \\
\hline NESLV & \multicolumn{2}{|c|}{ DIAGONAL SCALING MATRIX FOR F } \\
\hline NESLV & \multicolumn{2}{|c|}{$0.1000000000000 D+01 \quad 0.1000000000000 D+01$} \\
\hline NESLV & JACOBIAN FLAG & $\Leftrightarrow 1$ IF ANALYTIC JACOBIAN SUPPLIED \\
\hline NESLV & METHOD & $(=1$ IF TENSOR METHOD USED $)$ \\
\hline NESLV & GLOBAL STRATEGY & $(=0$ IF LINE SEARCH USED $)$ \\
\hline NESLV & ITERATION LIMIT & 150 \\
\hline NESLV & MACHINE EPSILON & $.2220446049250 D-15$ \\
\hline NESLV & STEP TOLERANCE & $.1000000000000 \mathrm{D}-08$ \\
\hline NESLV & GRADIENT TOLERANCE $=0$ & $.1000000000000 \mathrm{D}-04$ \\
\hline NESLV & FUNCTION TOLERANCE $=0$ & $.1000000000000 D-08$ \\
\hline NESLV & KAXIMUM STEP SIZE $=0$ & $.1000000000000 D+04$ \\
\hline NESLV & TRUST REG RADIUS & $.1000000000000 D+01$ \\
\hline RESULT & ITERATION $\mathrm{K}$ & \\
\hline RESULT & $X(X)$ & \\
\hline RESULT & $-0.1200000000000 D+01$ & $0.1000000000000 D+01$ \\
\hline RESULT & FUNCTION AT $X(\mathrm{~K})$ & \\
\hline RESULT & $0.1210000000000 D+02$ & \\
\hline RESULT & GRADIENT AT $X(K)$ & \\
\hline RESULT & $-0.1077999998579 D+03$ & $-0.4400000000000 D+02$ \\
\hline NLSTP & FUNCTION VALUE CLOSE I & O ZERO \\
\hline RESULT & ITERATION $K$ & \\
\hline RESULT & $X(K)$ & \\
\hline RESULT & $0.9999999997177 D+00$ & $0.9999999994362 \mathrm{D}+00$ \\
\hline RESULT & FUNCTION AT $X(K)$ & \\
\hline RESULT & $0.3986881344063 D-19$ & \\
\hline RESULT & GRADIENT AT $X(K)$ & \\
\hline RESULT & $-0.4270366293595 \mathrm{D}-09$ & $0.7237543951455 \mathrm{D}-10$ \\
\hline
\end{tabular}



FVEC:

If we now wish to solve the nonlinear least squares problem given by the following subroutine C
C
C The following is a subroutine for the Wood function.

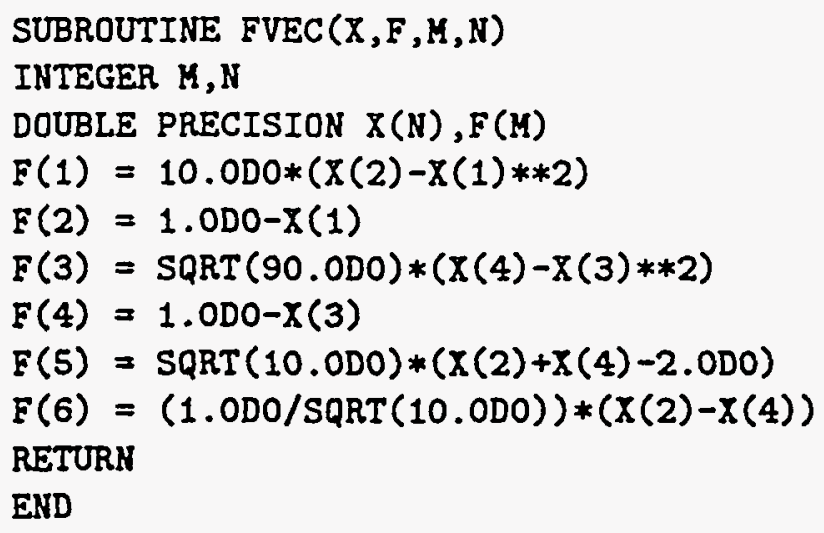

with the following input:

$M, N: 64$

XO: $\quad-30.0 D 0-10.0 D 0-30.0 D 0-10.0 D O$

GLOBAL: 1 (i.e. this is set after the call to DFAULT in the driver program) the output will be as follows:

\begin{tabular}{|c|c|c|c|}
\hline NESLV & \multicolumn{3}{|l|}{ TYPICAL $X$} \\
\hline NESLV & $0.1000000000000 D+01$ & $0.1000000000000 D+01$ & $0.1000000000000 D+01$ \\
\hline NESLV & \multicolumn{3}{|l|}{$0.1000000000000 D+01$} \\
\hline NESLV & \multicolumn{3}{|c|}{ DIAGONAL SCALING MATRIX FOR $\mathrm{X}$} \\
\hline NESLV & $0.1000000000000 D+01$ & $0.1000000000000 D+01$ & $0.1000000000000 D+01$ \\
\hline NESLV & $0.1000000000000 D+01$ & & \\
\hline NESLV & TYPICAL $F$ & & \\
\hline NESLV & $0.1000000000000 D+01$ & $0.1000000000000 D+01$ & $0.1000000000000 D+01$ \\
\hline NESLV & \multirow[t]{2}{*}{$0.1000000000000 D+01$} & $0.1000000000000 D+01$ & $0.1000000000000 D+01$ \\
\hline NESLV & & & \\
\hline NESLV & \multicolumn{3}{|c|}{ DIAGONAL SCALING MATRIX FOR F } \\
\hline NESLV & $0.1000000000000 D+01$ & $0.1000000000000 D+01$ & $0.1000000000000 D+01$ \\
\hline NESLV & \multirow{2}{*}{\multicolumn{2}{|c|}{$0.1000000000000 D+01$}} & $0.1000000000000 D+01$ \\
\hline NESLV & & & \\
\hline NESLV & JACOBIAN FLAG & \multicolumn{2}{|c|}{$=0$ ( $=1$ IF ANALYTIC JACOBIAN SUPPLIED) } \\
\hline NESLV & METHOD & \multicolumn{2}{|c|}{$=1(=1$ IF TENSOR METHOD USED $)$} \\
\hline NESLV & GLOBAL STRATEGY & \multicolumn{2}{|c|}{$=1 \quad(=0$ IF LINE SEARCH USED $)$} \\
\hline NESLV & ITERATION LIMIT & \multicolumn{2}{|l|}{$=150$} \\
\hline NESLV & MACHINE EPSILON & \multicolumn{2}{|l|}{$=0.2220446049250 \mathrm{D}-15$} \\
\hline NESLV & STEP TOLERANCE & \multicolumn{2}{|l|}{$=0.1000000000000 \mathrm{D}-08$} \\
\hline
\end{tabular}




\begin{tabular}{|c|c|c|c|}
\hline NESLV & GRADIENT TOLERANCE $=0$ & $0.1000000000000 D-04$ & \\
\hline NESLV & FUNCTION TOLERANCE $=0$ & $0.1000000000000 D-08$ & \\
\hline NESLV & MAXIMUM STEP SIZE $=0$ & $0.1000000000000 D+04$ & \\
\hline NESLV & TRUST REG RADIUS & $0.1000000000000 D+01$ & \\
\hline RESULT & ITERATION $\mathrm{K}$ & & \\
\hline RESULT & $X(K)$ & & \\
\hline RESULT & $-0.3000000000000 D+02$ & $-0.1000000000000 D+02$ & $-0.3000000000000 D+02$ \\
\hline RESULT & $-0.1000000000000 D+02$ & & \\
\hline RESULT & FUNCTION AT $X(K)$ & & \\
\hline RESULT & $0.7867288100000 \mathrm{D}+08$ & & \\
\hline RESULT & GRADIENT AT $X(K)$ & & \\
\hline RESULT & $-0.5460030962972 D+07$ & $-0.9122000000267 D+05$ & $-0.4914030950915 \mathrm{D}+07$ \\
\hline RESULT & $-0.8211996230035 D+05$ & & \\
\hline NLSTP & FUNCTION VALUE CLOSE I & TO ZERO & \\
\hline RESULT & ITERATION $\mathrm{K}$ & & \\
\hline RESULI & $X(K)$ & & \\
\hline RESULT & $0.1000000000000 D+01$ & $0.1000000000000 D+01$ & $0.1000000000000 D+01$ \\
\hline RESULT & $0.1000000000000 D+01$ & & \\
\hline RESULT & FUNCTION AT $X(K)$ & & \\
\hline RESULT & $0.2488861702651 D-26$ & & \\
\hline RESULT & GRADIENT AI $X(X)$ & & \\
\hline RESULT & $0.1178612771710 \mathrm{D}-11$ & $-0.6152522979420 D-12$ & $0.7212008821566 \mathrm{D}-12$ \\
\hline RESULT & $-0.3861688746554 D-12$ & & \\
\hline
\end{tabular}

\section{Test Results}

We have tested the TENSOLVE software package using the algorithms described above on a variety of nonsingular and singular problems. This section summarizes and discusses the test results.

In our tests, the package terminates successfully if the relative size of $\left(x_{+}-x_{c}\right)$ is less than macheps $s^{\frac{1}{2}}$, or $\left\|F\left(x_{+}\right)\right\|_{\infty}$ is less than macheps $s^{\frac{2}{3}}$. It terminates unsuccessfully if the iteration limit of 150 is exceeded. If the last global step fails to locate a point lower than $x_{c}$ in the line search or trust region global strategies, or the relative size of $J\left(x_{+}\right)^{T} F\left(x_{+}\right)$is less than macheps ${ }^{\frac{1}{3}}$, the method stops and reports this condition; this may indicate either success or failure. All our computations were performed on a Sun 4 computer in the Computer Science Department at the University of Colorado at Boulder, using double-precision arithmetic.

First we tested the software package on the set of nonlinear equations and nonlinear least squares problems in Moré, Garbow, and Hillstrom [9]. These problems all have nonsingular Jacobians at the solution with the exception of Powell's singular function. Then we created 
singular test problems as proposed in Schnabel and Frank [11] by modifying the nonsingular test problems of Moré, Garbow, and Hillstrom to the form

$$
\hat{F}(x)=F(x)-F^{\prime}\left(x_{*}\right) A\left(A^{T} A\right)^{-1} A^{T}\left(x-x_{*}\right),
$$

where $F(x)$ is the standard nonsingular test function, $x_{*}$ is its root or minimizer, and $A \in R^{m \times k}$ has full column rank with $1 \leq k \leq n$. Note that $x_{*}$ is a root or critical point of the modified problem, and rank $\hat{F}^{\prime}\left(x_{*}\right)=n-\operatorname{rank}(A)$. We used (10.1) to create two sets of singular problems, with $\hat{F}^{\prime}(x)$ having rank $n-1$ and $n-2$, respectively, by using

$$
A \in R^{m \times 1}, \quad A^{T}=(1,1, \ldots, 1),
$$

and

$$
A \in R^{m \times 2}, \quad A^{T}=\left[\begin{array}{cccccccc}
1 & 1 & 1 & 1 & \cdot & \cdot & \cdot & 1 \\
1 & -1 & 1 & -1 & \cdot & \cdot & \cdot & \pm 1
\end{array}\right]
$$

respectively.

We tested our tensor algorithm on 17 test functions for systems of nonlinear equations (also including 4 functions from [ 7 ] whose Jacobian at the solution $x_{*}$ is singular and are designated as Griewank functions) and 11 test functions for nonlinear least squares. Some of the test problems were run at various dimensions. All of these problems were also run with the standard method. The list of test problems is given in Appendix A; the detailed test results are given in [2].

Our computational results for the test problems whose Jacobians at the solution have ranks $n, n-1$, and $n-2$ are summarized in Tables 1 to 4 . In each of these tables, columns "Better" and "Worse" represent the number of times the tensor method was better and worse, respectively, than the standard method by more than one iteration. The "Tie" column represents the number of times the tensor and standard methods required within one iteration of each other. For each set of problems, we summarize the comparative costs of the tensor and standard methods using average ratios of two measures: iterations, and function evaluations. The average iteration ratio is the total number of iterations required by the tensor method over all the problems included, divided by the total number of iterations required by the standard method on the same problems. The same measure is used for the average function evaluation ratio. These average ratios include only problems that were successfully solved by both methods. We have excluded from the summary of statistics all cases where the tensor and standard methods converge to a different root, or to the same root as each other but not the singular root $x_{*}$ in the case of singular problems. However, the statistics for the "Better," "Worse," and "Tie" columns include the cases where only one of the two methods converges, and exclude the cases where both methods do not converge. The total number of problems that were solved by one method but not the other are given in the last two columns of each table.

In the test results obtained for both nonsingular and singular nonlinear equations problems, the tensor method virtually never is less efficient than the standard method and usually is more efficient. The improvement by the tensor method over the standard method with the same global strategy is substantial, averaging about $49 \%$ in iterations and $41 \%$ in function evaluations when the line search is used, and about $42 \%$ in iterations and $31 \%$ in function evaluations when the trust region is used, on the problems that are successfully solved by both methods. The improvement by the tensor method over the standard method is more dramatic on problems 
with small rank deficiency than on nonsingular problems, but is substantial in all cases. On rank $n-1$ problems, this is due in part to the tensor methods achieving 3 step Q-order $\frac{3}{2}$ convergence whereas the Newton's method is linearly convergent with constant $\frac{1}{2}$ [6].

The tensor method is also significantly more robust than the standard Newton-based method for the nonlinear equations test set. Over all the nonlinear equations test problems, 5 rank $n$ problems, 5 rank $n-1$ problems, and 8 rank $n-2$ problems were solved by the tensor method and not by the standard method when the line search was used, and 6 rank $n$ problems, 4 rank $n-1$ problems, and 4 rank $n-2$ problems were solved by the tensor method and not by the standard method when the trust region was used. On the other hand, only 1 rank $n$ problem was solved by the standard method and not by the tensor method when the line search was used, and only 1 rank $n$ problem was solved by the standard method and not by the tensor method when the trust region was used.

For the entire set of nonsingular and singular nonlinear least squares problems, the average improvement of the tensor method over the standard Gauss-Newton method also is substantial. Over the problems solved successfully by both methods, the improvement averages about $52 \%$ in iterations and $53 \%$ in function evaluations when the line search is used, and about $35 \%$ in iterations and $28 \%$ in function evaluations when the trust region is used.

The tensor method is also considerably more robust than the Gauss-Newton method for the nonlinear least squares test set, especially in the line search comparison. The tensor method solves several problems that the standard Gauss-Newton method does not, and the reverse never occurs. Over all the nonlinear least squares test problems, 4 rank $n$ problems, 2 rank $n-1$ problems, and 4 rank $n-2$ problems were solved by the tensor method and not by the standard Gauss-Newton method when the line search was used, and 3 rank $n$ problems, 1 rank $n-1$ problems, and 1 rank $n-2$ problems were solved by the tensor method and not by the standard Gauss-Newton method when the trust region was used. On the other hand, there were no. problems solved by the standard Gauss-Newton method and not by the tensor method when either the line search or the trust region was used.

A closer examination of the nonlinear least squares test results shows that the improvements by the tensor method are considerably larger for zero residual problems than for nonzero residual problems. The difference is most dramatic in the nonsingular case. Tables 5 and 6 show the average iteration and function evaluation ratios of the tensor method versus the Gauss-Newton method for zero and nonzero residual problems, respectively. The performance differences may be attributable to the fact that both the standard and tensor methods are linearly convergent on nonzero residual problems, but are more quickly convergent on zero residual problems.

The comparison between the line search methods and the trust region methods is very interesting, for both the standard and tensor methods. This is summarized in Tables $T$ and 8 . These tables show that on the average, the two-dimensional trust region approach often is more efficient than the line search method, especially on nonsingular problems. It is important to note, however, that the line search method is simpler to implement and to understand than the the two-dimensional trust region approach, and is appreciably faster in terms of CPU time on small, inexpensive problems where the complexity of the code becomes the dominant cost. It should also be noted that there is considerable variation in the comparative efficiency of the line search and trust region methods on individual problems and that either may be more efficient for a particular problem class. 
Perhaps a more important consideration in the general comparison of the line search and trust region methods, however, is that the two-dimensional trust region method solves considerably more of the test problems than the line search method. The advantage in robustness is particularly large in comparing line search and trust region versions of the standard methods; it is smaller but still significant in comparing tensor methods for nonlinear least squares, and insignificant in our tests of tensor methods for nonlinear equations. Over all the nonlinear equations test problems, 1 rank $n-1$ and 2 rank $n-2$ problems were solved by the trust region and not by the line search, whereas 1 rank $n$ problem and 1 rank $n-1$ problem were solved by the line search and not by the trust region, when the tensor method was used. On the other hand, when the Newton's method based code was used, 6 rank $n$ problems, 6 rank $n-1$ problems, and 5 rank $n-2$ problems were solved by the trust region and not by the line search, whereas only 1 rank $n$ problem, 1 rank $n-1$ problem, and 1 rank $n-2$ problem were solved by the line search and not by the trust region. Over all the nonlinear least squares test problems, 7 rank $n$ problems and 3 rank $n-2$ problems were solved by the trust region and not by the line search, whereas 4 rank $n$ problems were solved by the line search and not by the trust region, when the tensor method was used. When the standard Gauss-Newton method was used, 7 rank $n$ problems, 2 rank $n-1$ problems, and 8 rank $n-2$ problems were solved by the trust region and not by the line search, whereas only 1 rank $n-1$ problem and 1 rank $n-2$ problem were solved by the line search and not by the trust region. Thus, the trust region version seems to have a considerable advantage over the line search version in its robustness, although more when using the standard method than the tensor method. We note that the smaller average improvement of the tensor method over the standard method in the trust region cases (Tables 2 and 4) than the line search cases (Tables 1 and 3 ) is related to the difference in problem sets that are included in these statistics, because of the differing robustness of the line search and trust region methods.

Finally, we compared our tensor method with the NL2SOL package [5] on the set of nonlinear least squares problems used in [5] that is listed in Appendix B. The reason we were interested in making this comparison is that the NL2SOL method theoretically is superlinearly convergent on nonzero residual problems ([5]), whereas the tensor method of this paper, like Gauss-Newton methods, is only linearly convergent on nonzero residual problems. (This difference is related to NL2SOL using a quadratic model of $F(x)^{T} F(x)$ whereas the tensor and Gauss-Newton methods use models of $F(x)$.) The problems include a mixture of zero, small, and large residual problems.

Table 9 reports the comparative test results of the tensor method versus NL2SOL on this test set. The first row of Table 9 compares the tensor method using a line search with NL2SOL, whereas the second row compares the tensor method using a two-dimensional trust region with NL2SOL. (NL2SOL uses a trust region global strategy.) The table shows that on these test problems, the tensor method on the average is somewhat more efficient than NL2SOL, with an average improvement of about $58 \%$ in iterations and $29 \%$ in function evaluations when the line search is used, and about $24 \%$ in iterations and $7 \%$ in function evaluations when the trust region is used. (Note that the tensor method with line search is more efficient than the tensor method with trust region on this test set.) There is no difference in the robustness of the two packages of this test set; only 1 problem in the test set was solved by NL2SOL and not by the tensor method using either a line search or a trust region method, and only 1 problem was solved by the tensor method and not by NL2SOL. These limited results indicate that the tensor method appears to be quite competitive with NL2SOL for solving least squares problems. 
Table 1: Summary for Nonlinear Equations Test Problems using Line Search

\begin{tabular}{|c|c|c|c|c|c|c|c|}
\hline \multirow{3}{*}{$\begin{array}{l}\text { Rank } \\
F^{\prime}\left(x_{*}\right)\end{array}$} & \multicolumn{3}{|c|}{ Tensor } & \multirow{2}{*}{\multicolumn{2}{|c|}{$\begin{array}{l}\text { Average Ratio } \\
\text { Tensor/Newton }\end{array}$}} & \multirow{3}{*}{$\begin{array}{c}\text { Only } \\
\text { Newton } \\
\text { Solved }\end{array}$} & \multirow{3}{*}{$\begin{array}{l}\text { Only } \\
\text { Tensor } \\
\text { Solved }\end{array}$} \\
\hline & \multirow[t]{2}{*}{ Better } & \multirow[t]{2}{*}{ Worse } & \multirow[t]{2}{*}{ Tie } & & & & \\
\hline & & & & Itn & Feval & & \\
\hline$n$ & 25 & 2 & 13 & 0.60 & 0.69 & 1 & 5 \\
\hline$n-1$ & 24 & 0 & 8 & 0.48 & 0.53 & 0 & 5 \\
\hline$n-2$ & 27 & 1 & 5 & 0.46 & 0.56 & 0 & 8 \\
\hline
\end{tabular}

Table 2: Summary for Nonlinear Equations Test Problems Using Two-Dimensional Trust Region

\begin{tabular}{|c|c|c|c|c|c|c|c|}
\hline Rank & \multicolumn{3}{|c|}{ Tensor } & \multicolumn{2}{c|}{$\begin{array}{c}\text { Average Ratio } \\
\text { Tensor/Newton }\end{array}$} & $\begin{array}{c}\text { Only } \\
\text { Newton } \\
\text { Solved }\end{array}$ & $\begin{array}{c}\text { Only } \\
\text { Tensor } \\
\text { Solved }\end{array}$ \\
\cline { 2 - 6 } & Better & Worse & Tie & \multicolumn{2}{|c|}{ Tensol } \\
\cline { 5 - 7 } & & & & Itn & Feval & \\
\hline$n$ & 26 & 3 & 13 & 0.61 & 0.72 & 1 & 6 \\
$n-1$ & 24 & 1 & 9 & 0.49 & 0.63 & 0 & 4 \\
$n-2$ & 26 & 1 & 5 & 0.64 & 0.73 & 0 & 4 \\
\hline
\end{tabular}

Table 3: Summary for Nonlinear Least Squares Test Problems Using Line Search

\begin{tabular}{|c|c|c|c|c|c|c|c|}
\hline \multirow{3}{*}{$\begin{array}{l}\text { Rank } \\
F^{\prime}\left(x_{*}\right)\end{array}$} & \multicolumn{3}{|c|}{ Tensor } & \multirow{2}{*}{\multicolumn{2}{|c|}{$\begin{array}{c}\text { Average Ratio } \\
\text { Tensor/Gauss-Newton }\end{array}$}} & \multirow{3}{*}{$\begin{array}{c}\text { Only } \\
\text { Gauss- } 1 \text { Newton } \\
\text { Solved }\end{array}$} & \multirow{3}{*}{$\begin{array}{l}\text { Only } \\
\text { Tensor } \\
\text { Solved } \\
\end{array}$} \\
\hline & \multirow[t]{2}{*}{ Better } & \multirow[t]{2}{*}{ Worse } & \multirow[t]{2}{*}{ Tie } & & & & \\
\hline & & & & Itn & Feval & & \\
\hline$n$ & 20 & 1 & 8 & 0.52 & 0.51 & 0 & 4 \\
\hline$n-1$ & 18 & 0 & 8 & 0.45 & 0.41 & 0 & 2 \\
\hline$n-2$ & 28 & 0 & 5 & 0.48 & 0.48 & 0 & 4 \\
\hline
\end{tabular}

Table 4: Summary for Nonlinear Least Squares Test Problems Using Two-Dimensional Trust Region

\begin{tabular}{|c|c|c|c|c|c|c|c|}
\hline \multirow{3}{*}{$\begin{array}{l}\text { Rank } \\
F^{\prime}\left(x_{m}\right)\end{array}$} & \multicolumn{3}{|c|}{ Tensor } & \multirow{2}{*}{\multicolumn{2}{|c|}{$\begin{array}{c}\text { Average Ratio } \\
\text { Tensor/Gauss-Newton }\end{array}$}} & \multirow{3}{*}{$\begin{array}{c}\text { Only } \\
\text { Gauss-Newton } \\
\text { Solved }\end{array}$} & \multirow{3}{*}{$\begin{array}{l}\text { Only } \\
\text { Tensor } \\
\text { Solved } \\
\end{array}$} \\
\hline & \multirow[t]{2}{*}{ Better } & \multirow[t]{2}{*}{ Worse } & \multirow[t]{2}{*}{ Tie } & & & & \\
\hline & & & & Itn & Feval & & \\
\hline$n$ & 26 & 1 & 5 & 0.66 & 0.76 & 0 & 3 \\
\hline$n-1$ & 19 & 2 & 5 & 0.66 & 0.71 & 0 & 1 \\
\hline$n-2$ & 28 & 1 & 4 & 0.63 & 0.69 & 0 & 1 \\
\hline
\end{tabular}


Table 5: Average Ratios of the Tensor Method versus the Gauss-Newton Method on Zero Residual Problems for Line Search and Trust Region

\begin{tabular}{|c|c|c|c|c|}
\hline Rank & \multicolumn{2}{|c|}{ Line Search } & \multicolumn{2}{c|}{ Trust Region } \\
\hline$F^{\prime}\left(x_{*}\right)$ & Itn & Feval & Itn & Feval \\
\hline$n$ & 0.43 & 0.44 & 0.43 & 0.56 \\
$n-1$ & 0.41 & 0.37 & 0.64 & 0.62 \\
$n-2$ & 0.48 & 0.48 & 0.51 & 0.57 \\
\hline
\end{tabular}

Table 6: Average Ratios of the Tensor Method versus the Gauss-Newton Method on Nonzero Residual Problems for Line Search and Trust Region

\begin{tabular}{|c|c|c|c|c|}
\hline Rank & \multicolumn{2}{|c|}{ Line Search } & \multicolumn{2}{c|}{ Trust Region } \\
\hline$F^{\prime}\left(x_{*}\right)$ & Itn & Feval & Itn & Feval \\
\hline$n$ & 0.64 & 0.64 & 0.78 & 0.88 \\
$n-1$ & 0.48 & 0.45 & 0.67 & 0.79 \\
$n-2$ & 0.49 & 0.48 & 0.68 & 0.76 \\
\hline
\end{tabular}

Table 7: Average Ratios of Iterations and Function Evaluations of Newton with Trust Region versus Newton with Line Search and Tensor with Trust Region versus Tensor with Line Search for Nonlinear Equations

\begin{tabular}{|c|c|c|c|c|}
\hline Rank & \multicolumn{2}{|c|}{ Newton TR/LS } & \multicolumn{2}{c|}{ Tensor TR/LS } \\
\hline$F^{\prime}\left(x_{-}\right)$ & Itn & Feval & Itn & Feval \\
\hline$n$ & 0.80 & 0.84 & 0.70 & 0.57 \\
$n-1$ & 0.78 & 0.89 & 0.96 & 0.93 \\
$n-2$ & 0.86 & 0.93 & 0.92 & 0.94 \\
\hline
\end{tabular}

Table 8: Average Ratios of Iterations and Function Evaluations of Gauss-Newton with Trust Region versus Gauss-Newton with Line Search and Tensor with Trust Region versus Tensor with Line Search for Nonlinear Least Squares Problems

\begin{tabular}{|c|c|c|c|c|}
\hline Rank & \multicolumn{2}{|c|}{ Gauss-Newton TR/LS } & \multicolumn{2}{c|}{ Tensor TR/LS } \\
\hline$F^{\prime}\left(x_{*}\right)$ & Itn & Feval & Itn & Feval \\
\hline$n$ & 0.70 & 0.65 & 0.75 & 0.76 \\
$n-1$ & 0.72 & 0.71 & 1.05 & 1.09 \\
$n-2$ & 1.01 & 0.97 & 0.74 & 0.80 \\
\hline
\end{tabular}


Table 9: Comparison of Tensor Method with NL2SOL on the Nonlinear Equations and Nonlinear Least Squares Problems Listed in Table B-1

\begin{tabular}{|c|c|c|c|c|c|}
\hline Global strategy & \multicolumn{2}{|c|}{ Tensor versus NL2SOL } & \multicolumn{2}{c|}{ Average Ratio-Tensor/NL2SOL } \\
\hline & Better & Worse & Tie & Itn & Feval \\
\hline Tensor w/ LS & 25 & 8 & 2 & 0.42 & 0.71 \\
Tensor w/ TR & 24 & 9 & 2 & 0.76 & 0.93 \\
\hline
\end{tabular}

\section{References}

[1] A. Bouaricha, A Software Package for Solving Systems of Nonlinear Equations and Nonlinear Least Squares Problems Using Tensor Methods, M.S. thesis, Department of Computer Science, University of Colorado at Boulder, 1986.

[2] A. Bouaricha, Solving Large Sparse Systems of Nonlinear Equations and Nonlinear Least Squares Problems Using Tensor Methods on Sequential and Parallel Computers, Ph.D. thesis, Department of Computer Science, University of Colorado at Boulder, 1992.

[3] R. H. Byrd, R. B. Schnabel, and G. A. Shultz, Approximation Solution of the Trust Region Problem by Minimization over Two-Dimensional Subspaces, Mathematical Programming, 40 (1988), pp. 247-263.

[4] J. E. Dennis and R. B. Schnabel, Numerical Methods for Unconstrained Optimization and Nonlinear Equations, Prentice-Hall, Englewood Cliffs, N.J., 1983.

[5] J. E. Dennis, D. M. Gay, and R. E. Welsch, An Adaptive Nonlinear Least Squares Algorithm, ACM Trans. Math. Softw., 7 (1981), pp. 348-368.

[6] D. Feng, P. Frank, R. B. Schnabel, An Analysis of Tensor Methods for Nonlinear Equations, Technical Report CS-CS-729-94, Department of Computer Science, University of Colorado at Boulder, 1992.

[7] A. O. Griewank, Analysis and Modification of Newton's Method at Singularities, Ph.D. thesis, Australian National University, Canberra, 1980.

[8] J. J. Moré, The Levenberg-Marquardt Algorithm: Implementation and Theory, in Numerical Analysis, G. A. Watson, ed., Lecture Notes in Mathematics, vol. 630, Springer-Verlag, Berlin, 197T, pp. 105-116.

[9] J. J. Moré, B. S. Garbow, and K. E. Hillstrom, Testing Unconstrained Optimization Software, ACM Trans. Math. Softw., 7 (1981), pp. 17-41.

[10] M. J. D. Powell, A New Algorithm for Unconstrained Optimization, in Nonlinear Programming, J. B. Rosen, O.L. Mangasarian, and K. Ritter, eds., Academic Press, New York, pp. $1970,33-65$. 
[11] R. B. Schnabel and P. D. Frank, Tensor Methods for Nonlinear Equations, SIAM. J. Num. Anal., 21 (1984), pp. 815-843.

[12] R. B. Schnabel, J. E. Koontz, and B. E. Weiss, A Modular System of Algorithms of Unconstrained Minimization, ACM Trans. Math. Softw., 11 (1985), pp. 419-440. 


\section{Appendix A}

The columns in Tables A-1 and B-1 have the following meanings:

- Problem: name of the problem.

$-m$ : number of equations.

- $n$ : number of variables.

- NS: dimension of nullspace for Griewank's singular functions.

- OS: order of singularity for Griewank's singular functions.

Table A-1: List of Nonlinear Equations and Nonlinear Least Squares Test Problems Used in the Comparison of Tensor Method versus Standard Method

\begin{tabular}{|c|c|c|}
\hline Problem & \multicolumn{2}{|c|}{ Dimension } \\
\hline & $m$ & $n$ \\
\hline Brown almost linear & 10 & 10 \\
Broyden banded & 30 & 30 \\
Broyden tridiagonal & 30 & 30 \\
Chebyquad & 7 & 7 \\
Discrete boundary & 30 & 30 \\
Discrete integral & 10 & 10 \\
Helical valley & 3 & 3 \\
Powell singular & 4 & 4 \\
Rosenbrock & 2 & 2 \\
Trigonometric & 30 & 30 \\
Variable dimension & 10 & 10 \\
Watson & 31 & 31 \\
Wood gradient & 4 & 4 \\
NS = I OS =1 & 3 & 3 \\
NS = 2 OS =1 & 3 & 3 \\
NS = 1 OS = 2 & 3 & 3 \\
NS = 2 OS = 2 & 3 & 3 \\
\hline
\end{tabular}


Table A-1: List of Nonlinear Equations and Nonlinear Least Squares Test Problems Used in the Comparison of Tensor Method versus Standard Method (continued)

\begin{tabular}{|c|c|c|}
\hline Problem & \multicolumn{2}{|c|}{ Dimension } \\
\hline & $m$ & $n$ \\
\hline Wood & 6 & 4 \\
Variable dimension & 12 & 10 \\
Bard & 15 & 3 \\
Beale & 3 & 2 \\
Kowalik & 11 & 4 \\
Penalty1 & 11 & 10 \\
Penalty2 & 10 & 5 \\
Brown badly scaled & 3 & 2 \\
Gauss function & 15 & 3 \\
Brown and Dennis & 10 & 4 \\
Chebyquad & 8 & 4 \\
Chebyquad & 12 & 4 \\
Chebyquad & 16 & 4 \\
\hline
\end{tabular}

Appendix B

Table B-1: List of Nonlinear Equations and Nonlinear Least Squares Test Problems Used in the Comparison of Tensor Method versus ML2SOL

\begin{tabular}{|c|c|c|}
\hline Problem & \multicolumn{2}{|c|}{ Dimension } \\
\hline & $m$ & $n$ \\
\hline Rosenbrock & 2 & 2 \\
Helical Valley & 3 & 3 \\
Powell Singular & 4 & 4 \\
Wood & 6 & 4 \\
Beale & 3 & 2 \\
Box three-dimensional & 10 & 3 \\
Freudenstein and Roth & 2 & 2 \\
\hline
\end{tabular}


Table B-1: List of Nonlinear Equations and Nonlinear Least Squares Test Problems Used in the Comparison of Tensor Method versus NL.2SOL (continued)

\begin{tabular}{|c|c|c|}
\hline Problem & \multicolumn{2}{|c|}{ Dimension } \\
\hline & $m$ & $n$ \\
\hline Watson & 31 & 6 \\
Watson & 31 & 9 \\
Watson & 31 & 12 \\
Watson & 31 & 20 \\
Chebyquad & 8 & 8 \\
Bard & 15 & 3 \\
Jennrich and Sampson & 10 & 2 \\
Kowalik & 11 & 4 \\
Osborne 1 & 33 & 5 \\
Osborne 2 & 65 & 11 \\
\hline
\end{tabular}

